\title{
RANK RANGE TEST FOR EQUALITY OF DISPERSION
}

\author{
J. I. Odiase \\ Department of Mathematics, University of Benin, Nigeria
}

\begin{abstract}
This paper exploits the computational simplicity of the range of a set of data to formulate a twosample scale test called the Rank Range test. The performance of the test statistic is compared with other tests of scale. The exact distribution of the Rank Range test statistic is generated empirically through the unconditional permutation approach by actually obtaining all the distinct permutations of the ranks of the variates in an experiment; this exposes the trimodal nature of its probability distribution. The tables of exact critical values are produced.
\end{abstract}

Keywords: Algorithm, Exact test, Permutation test, Range, Rank test, Scale test.

\section{INTRODUCTION}

The proposed Rank Range test is a two-sample scale test and consists of independent random samples drawn from two populations. In twosample scale test, the population distributions are usually assumed to have the same location with different spreads or dispersion. However, if there is a difference in location, tests for difference in scale could be severely impaired (Neave and Worthington, 1988). In order to examine the performance of this proposed Rank Range test, a comparative study of the following nonparametric tests of scale is undertaken: Siegel-Tukey test, Mood test, Savage test for positive random variables, Ansari-Bradley test, Klotz test and Conover test (Lehmann, 1975; Conover, 1980; Gibbons,
1985) for expository descriptions of these tests.

The use of the asymptotic test with small sample sizes may yield an incorrect $\mathrm{p}$-value and therefore lead to a false acceptance or rejection of the null hypothesis (Mundry and Fischer, 1998). In order to avoid the use of incorrect p-values, the unconditional permutation approach is employed in this paper to arrive at the exact distribution of Rank Range test statistic for small sample sizes. The unconditional permutation approach is a statistical procedure that ensures that the exact distribution of a test statistic is obtained and that the resulting probability of a type I error is exactly $\alpha$ (Agresti, 1992; Good, 2000; Pesarin, 2001; Bagui and Bagui, 2004; Ernst, 2004; Odiase and Og- 
bonmwan, 2005a and b). Scheffe (1943) demonstrates that for a general class of problems, the permutation approach is the only possible method of truly constructing exact tests of significance. It is asymptotically as powerful as the best parametric test (Hoeffding, 1952).

In this paper, consideration is given to the exhaustive unconditional permutation of the ranks of the observations in a two-sample experiment to arrive at the exact distribution of the Rank Range statistic.

\section{MATERIALS AND METHODS}

The proposed nonparametric Rank Range $(R)$ test is attractive because of its computational simplicity. It is not sensitive to outliers, the null distribution depends only on sample size. The $R$ test is based on the reasoning that if two samples come from populations with the same median, the ranks of the observations in the sample with the greater variability will have a wider range. The null hypothesis is that there is no difference in spread between the two populations against the alternative hypothesis that there is some difference in spread between the two populations.

The purpose of this paper is to provide a theoretical basis for the $R$ test and provide empirically, via permutation, the exact critical values that will ensure that the probability of a type I error resulting from experiments involving the $R$ test is exactly $\alpha$.

In a two-sample problem composed of $X=\left\{x_{1}\right.$, $\left.\mathrm{x}_{2}, \ldots, x_{n 1}\right\}$ and $Y=\left\{\mathrm{y}_{1}, \mathrm{y}_{2}, \ldots, \mathrm{y}_{\mathrm{n} 2}\right\}$, arrange the combined samples in ascending order of magnitude and rank all the $N=n_{1}+n_{2}$ observations from 1 (smallest) to $N$ (largest). The $R$ test is defined as follows:

Given the layout of a two-sample experiment as

$$
\left(\begin{array}{cc}
x_{1} & y_{1} \\
x_{2} & y_{2} \\
\vdots & \vdots \\
x_{1 n_{1}} & y_{n_{2}}
\end{array}\right) \begin{aligned}
& \text { where } x_{i} \text { and } y_{i} \text { are the } i \text { th observa- } \\
& \text { tions of the independent random } \\
& \text { samples } X \text { and } Y \text { respectively. }
\end{aligned}
$$

$R=$ Difference between the Ranges of the Ranks of the two samples

$=\operatorname{Range}(X)-\operatorname{Range}(Y)$

$=\underset{1 \leq i, j \leq n_{1}}{\operatorname{Man}}\left|\operatorname{Rank}\left(x_{i}\right)-\operatorname{Rank}\left(x_{j}\right)\right|-\operatorname{Max}_{1 \leq i, j \leq n_{2}}\left|\operatorname{Rank}\left(y_{i}\right)-\operatorname{Rank}\left(y_{j}\right)\right|$

Let $\quad R_{X}={ }_{1 \leqslant i, j \leq n_{1}}^{\operatorname{Max}}\left|\operatorname{Rank}\left(x_{i}\right)-\operatorname{Rank}\left(x_{j}\right)\right| \quad$ and $\quad R_{Y}=\underset{1 \leq i, j \leq n_{2}}{\operatorname{Max}}\left|\operatorname{Rank}\left(y_{i}\right)-\operatorname{Rank}\left(y_{j}\right)\right|$ therefore, $R=R_{X}-R_{Y}$

The null hypothesis and the alternative hypotheses can be stated as follows:

Let $\mathrm{H}_{0}: F_{X}$ denote the CDF of $\mathrm{X}$ and assume that the CDF of Y satisfies $F_{Y}(y)=F_{X}(y / \theta)$, for $\theta>0$. The two-sided hypotheses are $H_{0}: \theta=1$ versus $H_{A}: \theta \neq 1$.

In order to have an insight into the distribution of $R$, the possible values of $R$ are now examined.

The maximum value of $R$ is attained when $R_{X}$ is maximum and $R_{Y}$ is minimum. That is,

$$
\begin{aligned}
& \operatorname{Max}(R)=\operatorname{Max}\left(R_{X}\right)-\operatorname{Min}\left(R_{Y}\right) \\
& \operatorname{But}, \operatorname{Max}\left(R_{X}\right)=\left(n_{1}+n_{2}\right)-1 \text { and } \\
& \operatorname{Min}\left(R_{Y}\right)=n_{2}-1
\end{aligned}
$$

Therefore, $\operatorname{Max}(R)=n_{1}$

The minimum value of $R$ is attained when $R_{X}$ is minimum and $R_{Y}$ is maximum. That is,

$\operatorname{Min}(R)=\operatorname{Min}\left(R_{X}\right)-\operatorname{Max}\left(R_{Y}\right)$

But $\operatorname{Min}\left(R_{X}\right)=n_{1}-1$ and $\operatorname{Max}\left(R_{Y}\right)=\left(n_{1}+n_{2}\right)-1$

Therefore, $\operatorname{Min}(R)=-n_{2}$.

$R_{X} \in\left\{n_{1}-1, n_{1}, \cdots, n_{1}+n_{2}-1\right\}$ and $n\left(R_{X}\right)=n_{2}+1$

where $n($.$) denotes cardinality.$

$R_{Y} \in\left\{n_{2}-1, n_{2}, \cdots, n_{1}+n_{2}-1\right\}$ and $n\left(R_{Y}\right)=n_{1}+1$

$R \in\left\{-n_{2},-n_{2}+1, \cdots,-1,0,1, \cdots, n_{1}-1, n_{1}\right\}$ and

$n(R)=n_{1}+n_{2}+1$ 
Permutation model for the Rank Range test

The idea of obtaining an exact test of significance through the permutation approach originated with Fisher (1935). The essential feature of the method is that all the distinct arrangements of the observations are considered, with the stipulation that all the permutations are equally likely under the null hypothesis. An exact test on the level of significance, $\alpha$, is constructed by choosing a proportion, $\alpha$, of the permutation as the critical region. The works of Conover (1980), Siegel and Castellan (1988), Headrick (2003), Bagui and Bagui (2004), Odiase and Ogbonmwan (2005a and b) are contributions to the quest for exact critical values.

Let the layout of the ranks $\left(r_{i j}\right)$ of the observations in a two-sample layout be

$$
L_{R}=\left(\begin{array}{cc}
r_{11} & r_{21} \\
r_{12} & r_{22} \\
\vdots & \vdots \\
r_{1 n_{1}} & r_{2 n_{2}}
\end{array}\right)
$$

Under the null hypothesis, $L_{R}$ is composed of $N$ $n_{1}+n_{2}$ independent and identically distributed random variables and hence conditioned on the observed data set. An exhaustive permutation of the ranks yields

$$
m=\left(\begin{array}{l}
N \\
n_{1}
\end{array}\right)=\left(\begin{array}{l}
N \\
n_{2}
\end{array}\right)=\frac{N !}{n_{1} ! n_{2} !}
$$

permutations of the $N$ ranks of the variates of two subsets of sizes $n_{l}$ and $n_{2}$, which are equally likely, each having the conditional probability $\mathrm{m}^{-1}$.

\section{Test Procedure}

Let $\pi_{1}, \pi_{2}, \ldots, \pi_{\mathrm{m}}$ be a set of all distinct permutations of the ranks of the data set in the experiment. The permutation test procedure is as follows:

1. Rank the combined observations of the experiment to obtain $L_{R}=\pi_{1}$ and compute the observed value of $R$ statistic to obtain $R_{1}=t_{0}$.
2. Obtain a distinct permutation $\pi_{i}$, of the ranks $\left(L_{R}\right)$ in Step 1.

3. Compute the $R$ statistic $R_{\mathrm{i}}=\mathrm{R}\left(\pi_{\mathrm{l}}\right)$, for permutation $\pi_{i}$ in Step 2, where $i>1$.

4. Perform Steps 2 and 3 for $i=2,3, \ldots, m$.

5. Construct the empirical cdf for the distinct values of $R$ and extract critical values.

$$
\begin{aligned}
& p_{0}=p\left(R \leq R_{i}\right)=\frac{1}{m} \sum_{i=1}^{m} \psi\left(R_{i}-t_{0}\right) \quad \text { where } \\
& \psi\left(R_{i}-t_{0}\right)= \begin{cases}1, & \text { if } R_{i} \leq t_{0} \\
0, & \text { if } R_{i}>t_{0}\end{cases}
\end{aligned}
$$

6. Under the empirical distribution, if $P_{0} \leq a$, reject the null hypothesis.

Let the initial configuration of the ranks of the variate in a two-sample experiment be $L_{R}$. A description of the algorithm for an exhaustive enumeration that produces the $m$ distinct permutations now follows.

Original arrangement/permutation:

$L_{R}$ is the original arrangement of the data of the experiment, this yields

$$
\left(\begin{array}{c}
\mathrm{n}_{1} \\
0
\end{array}\right)\left(\begin{array}{c}
\mathrm{n}_{2} \\
0
\end{array}\right)
$$

permutations.

Permutations involving exchange of one rank:

$r_{11} \leftrightarrow r_{21}, \mathrm{i}=1(1) \mathrm{n}_{2} \Rightarrow \mathrm{n}_{2}$ permutations

$r_{12} \leftrightarrow r_{2 i}, \quad \mathrm{i}=1(1) \mathrm{n}_{2} \Rightarrow \mathrm{n}_{2}$ permutations

...

$r_{1 n \mathrm{i}} \leftrightarrow r_{2 i}, \mathrm{i}=1(1) \mathrm{n}_{2} \Rightarrow \mathrm{n}_{2}$ permutations

Total: $\left(\begin{array}{c}\mathrm{n}_{1} \\ 1\end{array}\right)\left(\begin{array}{c}\mathrm{n}_{2} \\ 1\end{array}\right) \begin{aligned} & \text { permutations (one rank from } \\ & \text { first sample). }\end{aligned}$

Permutations involving exchange of two ranks:

$\left(\begin{array}{c}r_{1 s} \\ r_{1 t}\end{array}\right) \leftrightarrow\left(\begin{array}{c}r_{2 i} \\ r_{2 j}\end{array}\right) ; \quad \mathrm{s} \neq \mathrm{t}, \mathrm{i} \neq \mathrm{j}$ 
Total: $\quad\left(\begin{array}{c}\mathrm{n}_{1} \\ 2\end{array}\right)\left(\begin{array}{c}\mathrm{n}_{2} \\ 2\end{array}\right) \begin{aligned} & \text { permutations (two ranks } \\ & \text { from first sample) }\end{aligned}$

Permutations involving exchange of all ranks:

$\left(\begin{array}{c}r_{1 s} \\ r_{1 t} \\ \vdots \\ r_{1 u}\end{array}\right) \leftrightarrow\left(\begin{array}{c}r_{2 i} \\ r_{2 j} \\ \vdots \\ r_{2 k}\end{array}\right) ; \mathrm{s} \neq \mathrm{t} \neq \ldots \neq \mathrm{u} ; \mathrm{i} \neq \mathrm{j} \neq \ldots \neq \mathrm{k}$

Total: $\left(\begin{array}{c}n_{1} \\ \min \left(n_{1}, n_{2}\right)\end{array}\right)\left(\begin{array}{c}n_{2} \\ \min \left(n_{1}, n_{2}\right)\end{array}\right)$

permutations (switch all sample ranks).

The total number of distinct permutations of $L_{R}$ in a complete enumeration is

$\sum_{i=0}^{\min \left(n_{1}, n_{2}\right)}\left(\begin{array}{c}n_{1} \\ i\end{array}\right)\left(\begin{array}{c}n_{2} \\ i\end{array}\right) \begin{aligned} & \text {, see Odiase and Ogbonmwan } \\ & (2005 \mathrm{a}) .\end{aligned}$

The $R$ statistic is computed for each permutation in the complete enumeration of all the distinct permutations. The distribution of the test statistic is obtained by tabulating the distinct values of the statistic against their probabilities of occurrence in the complete enumeration. The two-sample

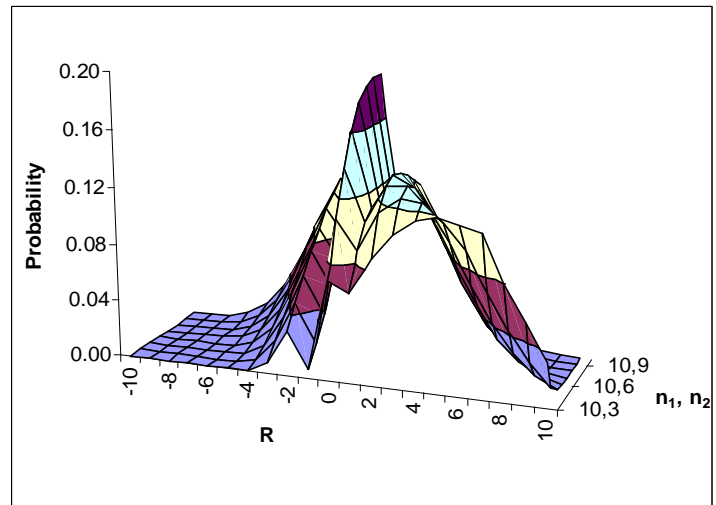

(a) $n_{1}=10, \quad 3 \leq n_{2} \leq 10$ unconditional permutation methodology and Algorithm described in Odiase and Ogbonmwan (2005a) is implemented for the $R$ statistic.

\section{RESULTS}

The trimodal distribution of $R$ statistic has modes at $E(R)-2, E(R)$ and $E(R)+2$. Figures 1(a)-(f) show the distribution of $R$ for different sample sizes. When $n_{l}$ is fixed (say 10 or 15) and $n_{2} \leq 15, R$ has the left-skewed distribution shown in Figures 1(a) and (b). When $n_{2}$ is fixed and $n_{1} \leq 15$ (say 3 or 6), $R$ has the right-skewed distribution shown in Figures 1 (c) and (d). When $n_{l}=n_{2} \leq 15, R$ has the symmetric distribution shown in Figure 1(e). The exact permutation distribution of $R$ for $n_{l}=n_{2}=15$ is in Figure 1(f).

The complete algorithm was implemented in Intel Visual Fortran. The exact critical values as obtained from the exhaustive unconditional permutation distribution of the $R$ test statistic for $n_{1}, n_{2} \leq$ 15 are presented in Table 1 (the two values in each cell represent the lower and upper critical values). The production of the statistical tables of $R$ for small sample sizes $\left(n_{1}, n_{2} \leq 15\right)$ removes the problem of non-availability of statistical tables often associated with the use of some nonparametric tests. With the provision of the statistical table for $R\left(n_{1}, n_{2} \leq 15\right)$, the t-test should be used to test $R$ for $15<n_{1}, n_{2}<30$, while the standard normal distribution is adopted for $n_{1} \geq 30$ or $n_{2} \geq 30$.

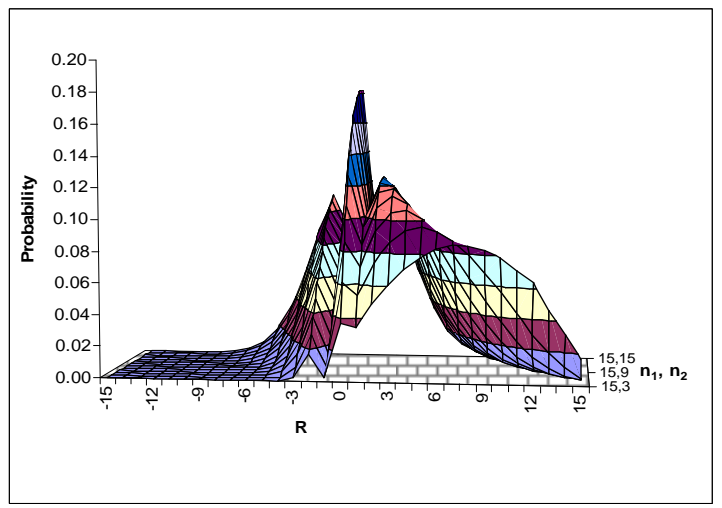

(b) $n_{1}=15, \quad 3 \leq n_{2} \leq 15$

144 Journal of Science and Technology, Vol. 29, No. 1, April, 2009 


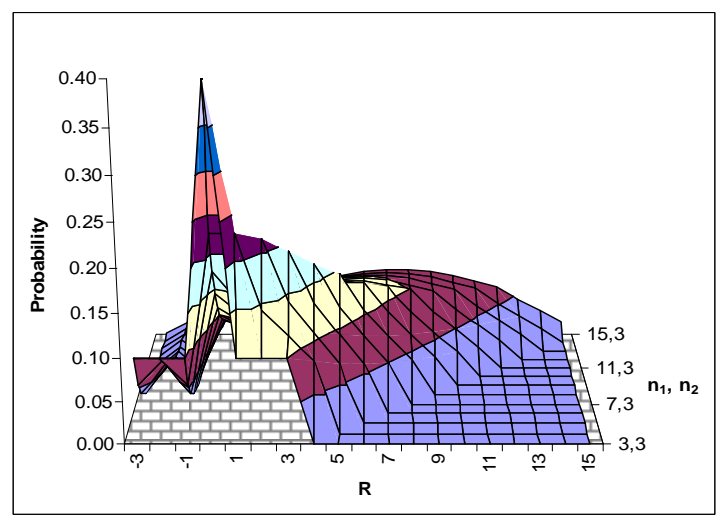

(c) $3 \leq n_{1} \leq 15, \quad n_{2}=3$

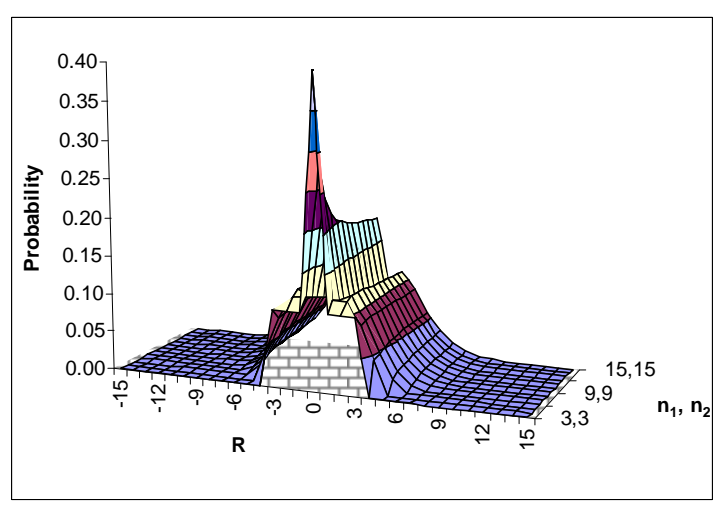

(e) $3 \leq n_{1}=n_{2} \leq 15$

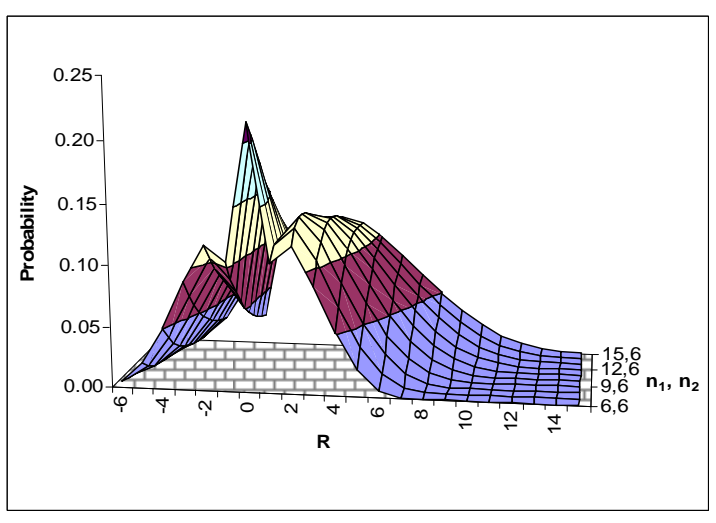

(d) $6 \leq n_{1} \leq 15, \quad n_{2}=6$

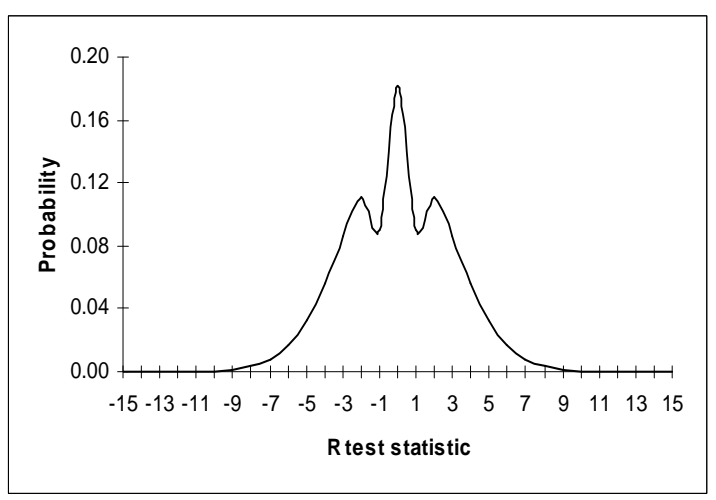

(f) $n_{1}=n_{2}=15$

Fig. 1: Probability distribution of $R$

\section{Comparative study of $\mathbf{R}$ statistic}

To demonstrate the ability of the $R$ statistic to properly test the null hypothesis of no difference in spread between two populations against the alternative hypothesis that there is some difference in spread between two populations, the following examples are considered.

\section{Example 1:}

The mouse data on survival times (Efron and Tibshirani, 1993)(see Table 2).
Sixteen mice were randomly assigned to a treatment group or a control group. Following a test surgery, their survival times, in days are examined to test the null hypothesis that variability in survival times in the control group is equal to the variability in survival times in treatment group. 
Table 1: Exact Critical values for Rank Range test

\begin{tabular}{|c|c|c|c|c|c|c|c|c|}
\hline \multicolumn{2}{|c|}{ Sample Size } & \multirow[t]{2}{*}{$\mathbf{R}_{\mathbf{0 . 9 0 0 0}}$} & \multirow[t]{2}{*}{$\mathbf{R}_{\mathbf{0 . 9 5 0 0}}$} & \multirow[t]{2}{*}{$\mathbf{R}_{\mathbf{0 . 9 7 5 0}}$} & \multirow[t]{2}{*}{$\mathbf{R}_{\mathbf{0 . 9 9 0 0}}$} & \multirow[t]{2}{*}{$\mathbf{R}_{\mathbf{0 . 9 9 5 0}}$} & \multirow[t]{2}{*}{$\mathbf{R}_{0.9975}$} & \multirow[t]{2}{*}{$\mathbf{R}_{\mathbf{0 . 9 9 9 0}}$} \\
\hline $\mathbf{n}_{1}$ & $\mathbf{n}_{2}$ & & & & & & & \\
\hline \multirow[t]{2}{*}{3} & 3 & -3 & & & & & & \\
\hline & & 3 & & & & & & \\
\hline \multirow[t]{2}{*}{4} & 3 & -2 & -3 & & & & & \\
\hline & & 3 & 4 & & & & & \\
\hline \multirow[t]{2}{*}{4} & 4 & -3 & -3 & & & & & \\
\hline & & 3 & 3 & & & & & \\
\hline \multirow[t]{2}{*}{5} & 3 & -2 & -2 & -3 & & & & \\
\hline & & 4 & 5 & 5 & & & & \\
\hline \multirow[t]{2}{*}{5} & 4 & -2 & -3 & -3 & & & & \\
\hline & & 4 & 4 & 5 & & & & \\
\hline \multirow[t]{2}{*}{5} & 5 & -3 & -4 & -4 & -5 & & & \\
\hline & & 3 & 4 & 4 & 5 & & & \\
\hline \multirow[t]{2}{*}{6} & 3 & -1 & -2 & -2 & & & & \\
\hline & & 5 & 6 & 6 & & & & \\
\hline \multirow[t]{2}{*}{6} & 4 & -2 & -3 & -3 & -4 & & & \\
\hline & & 4 & 5 & 5 & 6 & & & \\
\hline \multirow[t]{2}{*}{6} & 5 & -3 & -3 & -4 & -4 & -5 & & \\
\hline & & 4 & 4 & 5 & 6 & 6 & & \\
\hline \multirow[t]{2}{*}{6} & 6 & -3 & -4 & -5 & -5 & -6 & -6 & \\
\hline & & 3 & 4 & 5 & 5 & 6 & 6 & \\
\hline \multirow[t]{2}{*}{7} & 3 & 0 & -2 & -2 & -3 & & & \\
\hline & & 6 & 7 & 7 & 7 & & & \\
\hline \multirow[t]{2}{*}{7} & 4 & -2 & -2 & -3 & -3 & -4 & & \\
\hline & & 5 & 6 & 6 & 7 & 7 & & \\
\hline \multirow[t]{2}{*}{7} & 5 & -2 & -3 & -4 & -4 & -5 & -5 & \\
\hline & & 4 & 5 & 6 & 6 & 7 & 7 & \\
\hline 7 & 6 & -3 & -4 & -4 & -5 & -5 & -6 & -6 \\
\hline & & 4 & 5 & 5 & 6 & 6 & 7 & 7 \\
\hline 7 & 7 & -3 & -4 & -5 & -6 & -6 & -6 & -7 \\
\hline & & 3 & 4 & 5 & 6 & 6 & 6 & 7 \\
\hline 8 & 3 & 0 & -2 & -2 & -3 & & & \\
\hline & & 7 & 7 & 8 & 8 & & & \\
\hline 8 & 4 & -1 & -2 & -3 & -3 & -4 & & \\
\hline & & 6 & 7 & 7 & 8 & 8 & & \\
\hline 8 & 5 & -2 & -3 & -3 & -4 & -4 & -5 & \\
\hline & & 5 & 6 & 6 & 7 & 8 & 8 & \\
\hline 8 & 6 & -3 & -3 & -4 & -5 & -5 & -5 & -6 \\
\hline & & 4 & 5 & 6 & 7 & 7 & 7 & 8 \\
\hline 8 & 7 & -3 & -4 & -4 & -5 & -6 & -6 & -6 \\
\hline & & 4 & 5 & 5 & 6 & 7 & 7 & 8 \\
\hline 8 & 8 & -3 & -4 & -5 & -6 & -6 & -7 & -7 \\
\hline & & 3 & 4 & 5 & 6 & 6 & 7 & 7 \\
\hline 9 & 3 & 0 & -1 & -2 & -2 & -3 & & \\
\hline & & 8 & 8 & 9 & 9 & 9 & & \\
\hline 9 & 4 & -1 & -2 & -3 & -3 & -3 & -4 & \\
\hline & & 6 & 7 & 8 & 9 & 9 & 9 & \\
\hline
\end{tabular}

146 Journal of Science and Technology, Vol. 29, No. 1, April, 2009 
Table 1: Exact Critical values for Rank Range test (Contd.)

\begin{tabular}{|c|c|c|c|c|c|c|c|c|}
\hline \multicolumn{2}{|c|}{ Sample Size } & \multirow[t]{2}{*}{$\mathbf{R}_{\mathbf{0 . 9 0 0 0}}$} & \multirow[t]{2}{*}{$\mathbf{R}_{\mathbf{0 . 9 5 0 0}}$} & \multirow[t]{2}{*}{$\mathbf{R}_{\mathbf{0 . 9 7 5 0}}$} & \multirow[t]{2}{*}{$\mathbf{R}_{\mathbf{0 . 9 9 0 0}}$} & \multirow[t]{2}{*}{$\mathbf{R}_{\mathbf{0 . 9 9 5 0}}$} & \multirow[t]{2}{*}{$\mathbf{R}_{\mathbf{0 . 9 9 7 5}}$} & \multirow[t]{2}{*}{$\mathbf{R}_{\mathbf{0 . 9 9 9 0}}$} \\
\hline $\mathrm{n}_{1}$ & $\mathbf{n}_{2}$ & & & & & & & \\
\hline \multirow[t]{2}{*}{9} & 5 & -2 & -3 & -3 & -4 & -4 & -4 & -5 \\
\hline & & 6 & 6 & 7 & 8 & 8 & 9 & 9 \\
\hline \multirow[t]{2}{*}{9} & 6 & -2 & -3 & -4 & -4 & -5 & -5 & -5 \\
\hline & & 5 & 6 & 7 & 7 & 8 & 8 & 9 \\
\hline \multirow[t]{2}{*}{9} & 7 & -3 & -3 & -4 & -5 & -5 & -6 & -6 \\
\hline & & 4 & 5 & 6 & 7 & 7 & 8 & 8 \\
\hline \multirow[t]{2}{*}{9} & 8 & -3 & -4 & -5 & -5 & -6 & -6 & -7 \\
\hline & & 4 & 5 & 6 & 6 & 7 & 7 & 8 \\
\hline \multirow[t]{2}{*}{9} & 9 & -4 & -4 & -5 & -6 & -7 & -7 & -8 \\
\hline & & 4 & 4 & 5 & 6 & 7 & 7 & 8 \\
\hline \multirow[t]{2}{*}{10} & 3 & 0 & 0 & -2 & -2 & -3 & & \\
\hline & & 8 & 9 & 10 & 10 & 10 & & \\
\hline \multirow[t]{2}{*}{10} & 4 & 0 & -2 & -2 & -3 & -3 & -4 & -4 \\
\hline & & 7 & 8 & 9 & 9 & 10 & 10 & 10 \\
\hline \multirow[t]{2}{*}{10} & 5 & -2 & -2 & -3 & -4 & -4 & -4 & -5 \\
\hline & & 6 & 7 & 8 & 9 & 9 & 10 & 10 \\
\hline \multirow[t]{2}{*}{10} & 6 & -2 & -3 & -3 & -4 & -5 & -5 & -5 \\
\hline & & 5 & 6 & 7 & 8 & 9 & 9 & 10 \\
\hline \multirow[t]{2}{*}{10} & 7 & -2 & -3 & -4 & -5 & -5 & -6 & -6 \\
\hline & & 5 & 6 & 7 & 8 & 8 & 9 & 9 \\
\hline \multirow[t]{2}{*}{10} & 8 & -3 & -4 & -4 & -5 & -6 & -6 & -7 \\
\hline & & 4 & 5 & 6 & 7 & 8 & 8 & 9 \\
\hline \multirow[t]{2}{*}{10} & 9 & -3 & -4 & -5 & -6 & -6 & -7 & -7 \\
\hline & & 4 & 5 & 6 & 7 & 7 & 8 & 8 \\
\hline \multirow[t]{2}{*}{10} & 10 & -4 & -5 & -5 & -6 & -7 & -7 & -8 \\
\hline & & 4 & 5 & 5 & 6 & 7 & 7 & 8 \\
\hline \multirow[t]{2}{*}{11} & 3 & 1 & 0 & -2 & -2 & -3 & & \\
\hline & & 9 & 10 & 11 & 11 & 11 & & \\
\hline 11 & 4 & 0 & -2 & -2 & -3 & -3 & -3 & -4 \\
\hline & & 8 & 9 & 10 & 10 & 11 & 11 & 11 \\
\hline 11 & 5 & -1 & -2 & -3 & -3 & -4 & -4 & -4 \\
\hline & & 7 & 8 & 9 & 10 & 10 & 10 & 11 \\
\hline 11 & 6 & -2 & -3 & -3 & -4 & -4 & -5 & -5 \\
\hline & & 6 & 7 & 8 & 9 & 9 & 10 & 10 \\
\hline 11 & 7 & -2 & -3 & -4 & -4 & -5 & -5 & -6 \\
\hline & & 5 & 6 & 7 & 8 & 9 & 9 & 10 \\
\hline 11 & 8 & -3 & -3 & -4 & -5 & -5 & -6 & -6 \\
\hline & & 5 & 6 & 7 & 8 & 8 & 9 & 9 \\
\hline 11 & 9 & -3 & -4 & -5 & -5 & -6 & -6 & -7 \\
\hline & & 4 & 5 & 6 & 7 & 8 & 8 & 9 \\
\hline 11 & 10 & -3 & -4 & -5 & -6 & -7 & -7 & -8 \\
\hline & & 4 & 5 & 6 & 7 & 7 & 8 & 9 \\
\hline 11 & 11 & -4 & -5 & -5 & -6 & -7 & -8 & -8 \\
\hline & & 4 & 5 & 5 & 6 & 7 & 8 & 8 \\
\hline
\end{tabular}


Table 1: Exact Critical values for Rank Range test (Contd.)

\begin{tabular}{|c|c|c|c|c|c|c|c|c|}
\hline \multicolumn{2}{|c|}{ Sample Size } & \multirow[t]{2}{*}{$\mathbf{R}_{\mathbf{0 . 9 0 0 0}}$} & \multirow[t]{2}{*}{$\mathbf{R}_{\mathbf{0 . 9 5 0 0}}$} & \multirow[t]{2}{*}{$\mathbf{R}_{\mathbf{0 . 9 7 5 0}}$} & \multirow[t]{2}{*}{$\mathbf{R}_{\mathbf{0 . 9 9 0 0}}$} & \multirow[t]{2}{*}{$\mathbf{R}_{\mathbf{0 . 9 9 5 0}}$} & \multirow[t]{2}{*}{$\mathbf{R}_{0.9975}$} & \multirow[t]{2}{*}{$\mathbf{R}_{0.9990}$} \\
\hline $\mathrm{n}_{1}$ & $\mathbf{n}_{2}$ & & & & & & & \\
\hline \multirow[t]{2}{*}{12} & 3 & 1 & 0 & -1 & -2 & -2 & -3 & \\
\hline & & 10 & 11 & 11 & 12 & 12 & 12 & \\
\hline \multirow[t]{2}{*}{12} & 4 & 0 & -2 & -2 & -3 & -3 & -3 & -4 \\
\hline & & 9 & 10 & 10 & 11 & 12 & 12 & 12 \\
\hline \multirow[t]{2}{*}{12} & 5 & -1 & -2 & -3 & -3 & -4 & -4 & -4 \\
\hline & & 7 & 9 & 9 & 10 & 11 & 11 & 12 \\
\hline \multirow[t]{2}{*}{12} & 6 & -2 & -2 & -3 & -4 & -4 & -5 & -5 \\
\hline & & 7 & 8 & 9 & 10 & 10 & 11 & 11 \\
\hline \multirow[t]{2}{*}{12} & 7 & -2 & -3 & -3 & -4 & -5 & -5 & -6 \\
\hline & & 6 & 7 & 8 & 9 & 10 & 10 & 11 \\
\hline \multirow[t]{2}{*}{12} & 8 & -2 & -3 & -4 & -5 & -5 & -6 & -6 \\
\hline & & 5 & 6 & 7 & 8 & 9 & 10 & 10 \\
\hline \multirow[t]{2}{*}{12} & 9 & -3 & -4 & -4 & -5 & -6 & -6 & -7 \\
\hline & & 5 & 6 & 7 & 8 & 8 & 9 & 10 \\
\hline \multirow[t]{2}{*}{12} & 10 & -3 & -4 & -5 & -6 & -6 & -7 & -7 \\
\hline & & 4 & 5 & 6 & 7 & 8 & 9 & 9 \\
\hline \multirow[t]{2}{*}{12} & 11 & -3 & -4 & -5 & -6 & -7 & -7 & -8 \\
\hline & & 4 & 5 & 6 & 7 & 8 & 8 & 9 \\
\hline \multirow[t]{2}{*}{12} & 12 & -4 & -5 & -6 & -7 & -7 & -8 & -9 \\
\hline & & 4 & 5 & 6 & 7 & 7 & 8 & 9 \\
\hline \multirow[t]{2}{*}{13} & 3 & 1 & 0 & -2 & -2 & -2 & -3 & \\
\hline & & 11 & 12 & 12 & 13 & 13 & 13 & \\
\hline \multirow[t]{2}{*}{13} & 4 & 0 & -1 & -2 & -3 & -3 & -3 & -4 \\
\hline & & 9 & 10 & 11 & 12 & 13 & 13 & 13 \\
\hline \multirow[t]{2}{*}{13} & 5 & 0 & -2 & -2 & -3 & -3 & -4 & -4 \\
\hline & & 8 & 9 & 10 & 11 & 12 & 12 & 13 \\
\hline 13 & 6 & -1 & -2 & -3 & -4 & -4 & -4 & -5 \\
\hline & & 7 & 8 & 9 & 10 & 11 & 12 & 12 \\
\hline 13 & 7 & -2 & -3 & -3 & -4 & -4 & -5 & -5 \\
\hline & & 6 & 7 & 8 & 10 & 10 & 11 & 12 \\
\hline 13 & 8 & -2 & -3 & -4 & -4 & -5 & -5 & -6 \\
\hline & & 6 & 7 & 8 & 9 & 10 & 10 & 11 \\
\hline 13 & 9 & -3 & -3 & -4 & -5 & -5 & -6 & -7 \\
\hline & & 5 & 6 & 7 & 8 & 9 & 10 & 10 \\
\hline 13 & 10 & -3 & -4 & -4 & -5 & -6 & -7 & -7 \\
\hline & & 5 & 6 & 7 & 8 & 9 & 9 & 10 \\
\hline 13 & 11 & -3 & -4 & -5 & -6 & -6 & -7 & -8 \\
\hline & & 4 & 5 & 6 & 7 & 8 & 9 & 10 \\
\hline 13 & 12 & -3 & -4 & -5 & -6 & -7 & -8 & -8 \\
\hline & & 4 & 5 & 6 & 7 & 8 & 8 & 9 \\
\hline 13 & 13 & -4 & -5 & -6 & -7 & -7 & -8 & -9 \\
\hline & & 4 & 5 & 6 & 7 & 7 & 8 & 9 \\
\hline 14 & 3 & 1 & 0 & -1 & -2 & -2 & -3 & \\
\hline & & 12 & 13 & 13 & 14 & 14 & 14 & \\
\hline 14 & 4 & 0 & 0 & -2 & -2 & -3 & -3 & -3 \\
\hline & & 10 & 11 & 12 & 13 & 13 & 14 & 14 \\
\hline
\end{tabular}

148 Journal of Science and Technology, Vol. 29, No. 1, April, 2009 
Table 1: Exact Critical values for Rank Range test (Contd.)

\begin{tabular}{|c|c|c|c|c|c|c|c|c|}
\hline \multicolumn{2}{|c|}{ Sample Size } & \multirow[t]{2}{*}{$\mathbf{R}_{\mathbf{0 . 9 0 0 0}}$} & \multirow[t]{2}{*}{$\mathbf{R}_{0.9500}$} & \multirow[t]{2}{*}{$\mathbf{R}_{\mathbf{0 . 9 7 5 0}}$} & \multirow[t]{2}{*}{$\mathbf{R}_{0.9900}$} & \multirow[t]{2}{*}{$\mathbf{R}_{0.9950}$} & \multirow[t]{2}{*}{$\mathbf{R}_{0.9975}$} & \multirow[t]{2}{*}{$\mathbf{R}_{0.9990}$} \\
\hline $\mathrm{n}_{1}$ & $\mathbf{n}_{2}$ & & & & & & & \\
\hline \multirow[t]{2}{*}{14} & 5 & 0 & -2 & -2 & -3 & -3 & -4 & -4 \\
\hline & & 9 & 10 & 11 & 12 & 13 & 13 & 14 \\
\hline \multirow[t]{2}{*}{14} & 6 & -1 & -2 & -3 & -3 & -4 & -4 & -5 \\
\hline & & 8 & 9 & 10 & 11 & 12 & 12 & 13 \\
\hline \multirow[t]{2}{*}{14} & 7 & -2 & -2 & -3 & -4 & -4 & -5 & -5 \\
\hline & & 7 & 8 & 9 & 10 & 11 & 12 & 12 \\
\hline \multirow[t]{2}{*}{14} & 8 & -2 & -3 & -4 & -4 & -5 & -5 & -6 \\
\hline & & 6 & 7 & 8 & 10 & 10 & 11 & 12 \\
\hline \multirow[t]{2}{*}{14} & 9 & -2 & -3 & -4 & -5 & -5 & -6 & -6 \\
\hline & & 6 & 7 & 8 & 9 & 10 & 10 & 11 \\
\hline \multirow[t]{2}{*}{14} & 10 & -3 & -4 & -4 & -5 & -6 & -6 & -7 \\
\hline & & 5 & 6 & 7 & 8 & 9 & 10 & 11 \\
\hline \multirow[t]{2}{*}{14} & 11 & -3 & -4 & -5 & -6 & -6 & -7 & -7 \\
\hline & & 5 & 6 & 5 & 8 & 9 & 9 & 10 \\
\hline \multirow[t]{2}{*}{14} & 12 & -3 & -4 & -5 & -6 & -7 & -7 & -8 \\
\hline & & 4 & 5 & 6 & 7 & 8 & 9 & 10 \\
\hline \multirow[t]{2}{*}{14} & 13 & -4 & -4 & -5 & -6 & -7 & -8 & -8 \\
\hline & & 4 & 5 & 6 & 7 & 8 & 9 & 9 \\
\hline \multirow[t]{2}{*}{14} & 14 & -4 & -5 & -6 & -7 & -8 & -8 & -9 \\
\hline & & 4 & 5 & 6 & 7 & 8 & 8 & 9 \\
\hline \multirow[t]{2}{*}{15} & 3 & 2 & 0 & 0 & -2 & -2 & -2 & \\
\hline & & 12 & 13 & 14 & 15 & 15 & 15 & \\
\hline \multirow[t]{2}{*}{15} & 4 & 0 & 0 & -2 & -2 & -3 & -3 & -3 \\
\hline & & 11 & 12 & 13 & 14 & 14 & 15 & 15 \\
\hline \multirow[t]{2}{*}{15} & 5 & 0 & -2 & -2 & -3 & -3 & -4 & -4 \\
\hline & & 9 & 11 & 12 & 13 & 13 & 14 & 14 \\
\hline 15 & 6 & -1 & -2 & -3 & -3 & -4 & -4 & -5 \\
\hline & & 8 & 9 & 11 & 12 & 12 & 13 & 14 \\
\hline 15 & 7 & -2 & -2 & -3 & -4 & -4 & -5 & -5 \\
\hline & & 7 & 9 & 10 & 11 & 12 & 12 & 13 \\
\hline 15 & 8 & -2 & -3 & -3 & -4 & -5 & -5 & -6 \\
\hline & & 7 & 8 & 9 & 10 & 11 & 12 & 12 \\
\hline 15 & 9 & -2 & -3 & -4 & -5 & -5 & -6 & -6 \\
\hline & & 6 & 7 & 8 & 9 & 10 & 12 & 12 \\
\hline 15 & 10 & -2 & -3 & -4 & -5 & -6 & -6 & -7 \\
\hline & & 5 & 7 & 8 & 9 & 10 & 10 & 11 \\
\hline 15 & 11 & -3 & -4 & -4 & -5 & -6 & -7 & -7 \\
\hline & & 5 & 6 & 7 & 8 & 9 & 10 & 11 \\
\hline 15 & 12 & -3 & -4 & -5 & -6 & -6 & -7 & -8 \\
\hline & & 5 & 6 & 7 & 8 & 9 & 9 & 10 \\
\hline 15 & 13 & -3 & -4 & -5 & -6 & -7 & -7 & -8 \\
\hline & & 4 & 5 & 6 & 8 & 8 & 9 & 10 \\
\hline 15 & 14 & -4 & -5 & -5 & -7 & -7 & -8 & -9 \\
\hline & & 4 & 5 & 6 & 7 & 8 & 9 & 10 \\
\hline 15 & 15 & -4 & -5 & -6 & -7 & -8 & -8 & -9 \\
\hline & & 4 & 5 & 6 & 7 & 8 & 8 & 9 \\
\hline
\end{tabular}


Table 2: Test of the mouse data

\begin{tabular}{lllc}
\hline \multicolumn{1}{c}{ Test Statistic } & \multicolumn{1}{c}{ Test value } & p-value & $\begin{array}{c}\text { Decision on Null Hypothesis } \\
(\mathrm{a}=0.05)\end{array}$ \\
\hline Siegel-Tukey & 86 & 0.1755 & Accept \\
Mood & 156.25 & 0.1944 & Accept \\
Savage & -1.4378 & 0.2292 & Accept \\
Ansari-Bradley & 46 & 0.1477 & Accept \\
Klotz & 5.3016 & 0.2425 & Accept \\
Conover & 576 & 0.0568 & Accept \\
R & 0 & 0.4771 & Accept \\
\hline
\end{tabular}

Table 3: Test of the streamflow and precipitation

\begin{tabular}{lllc}
\hline \multicolumn{1}{c}{ Test Statistic } & \multicolumn{1}{c}{ Test value } & p-value & $\begin{array}{c}\text { Decision on Null Hypothesis } \\
(\mathrm{a}=0.05)\end{array}$ \\
\hline Siegel-Tukey & 74 & 0.2707 & Accept \\
Mood & 253 & 0.2166 & Accept \\
Savage & 1.1197 & 0.2943 & Accept \\
Ansari-Bradley & 40 & 0.3281 & Accept \\
Klotz & 8.3916 & 0.1519 & Accept \\
Conover & 1271 & 0.0449 & Reject \\
R & 3 & 0.2304 & Accept \\
\hline
\end{tabular}

\section{Example 2:}

Daily accumulated streamflow and precipitation (Gastwirth and Mahmoud 1986)

Daily accumulated streamflow and precipitation (in inches) for two U.S. Geological Survey stations in Colorado. Test the null hypothesis that the variability of streamflow and precipitation in the two Stations is the same (see Table 3).

\section{DISCUSSION}

This paper exploits the computational simplicity of the range of a set of data to formulate a twosample scale test called the Rank Range test. The exact distribution of the proposed Rank Range test statistic is generated empirically through the unconditional permutation approach by actually obtaining all the distinct permutations of the ranks of the variates in an experiment.

The distributions in Figure 1 clearly reveal that $R$ statistic has a trimodal distribution. It is symmet- ric for $n_{l}=n_{2}$ and skewed otherwise. Since this paper provides exact critical values for the group sample size up to $\max \left(n_{1}, n_{2}\right)=15$, permutation procedure should be implemented for $n_{1}, n_{2}>15$ to test $R$. The critical values of the $R$ test statistic in Table 1 are obtained from the enumeration of all the distinct permutations of the ranks of the variates in an experiment. These critical values are exact and therefore ensures that the probability of a type I error in decisions arising from the use of the Rank Range test is exactly $\alpha$.

The introduction of the Rank Range test statistic makes it possible to use the range of a set of values as a test statistic. The value of the test statistic can easily be computed for a given data set. For small sample sizes, $R$ can be computed simply by inspection of the data set.

\section{CONCLUSION}

The computational simplicity of the range of a set of data is exploited to formulate a two-sample 
scale test called the Rank Range test. The performance of the Rank Range test statistic is compared with other existing tests of scale and found to yield the same results in all the cases considered. The exact distribution of the Rank Range test statistic is generated empirically through the unconditional permutation approach and the probability distribution is found to be trimodal. The table of exact critical values is produced for the group sample size up to $\max \left(n_{1}, n_{2}\right)=15$.

\section{REFERENCES}

Agresti, A. (1992). A survey of exact inference for contingency tables, Statistical Science 7: 131-177.

Bagui, S. and Bagui, S. (2004). An algorithm and code for computing exact critical values for the Kruskal-Wallis nonparametric one-way ANOVA. Journal of Modern Applied Statistical Methods 3: 498-503.

Conover, W. J. (1980). Practical nonparametric statistics (2nd ed.). John Wiley and Sons, New York.

Efron, B. and Tibshirani, R.J. (1993). An introduction to the bootstrap. Chapman and Hall, New York.

Ernst M. D. (2004). Permutation methods: A basis for exact inference. Statistical Science, 19: 676-685.

Fisher, R. A. (1935). The design of experiments. Oliver and Boyd, Edinburgh

Gastwirth, J. L. and Mahmoud, H. (1986). An efficient robust nonparametric test for scale change for data from a gamma distribution. Technometrics, 28(1): p. 83

Gibbons, J. D. (1985). Nonparametric statistical inference (2nd ed.). Marcel Dekker, New York.

Good, P. (2000). Permutation tests: a practical guide to resampling methods for testing hypotheses (2nd edition). Springer Verlag, New York.
Headrick, T. C. (2003). An algorithm for generating exact critical values for the KruskalWallis one-way ANOVA. Journal of Modern Applied Statistical Methods 2: 268-271.

Hoeffding, W. (1952). Large sample power of tests based on permutations of observations. The Annals of Mathematical Statistics, 23: 169-192.

Lehmann, E. L. (1975). Nonparametrics: Statistical methods based on ranks. Holden Day, San Francisco.

Mundry R. and Fischer, J. (1998). Use of statistical programs for nonparametric tests of small samples often leads to incorrect $\mathrm{P}$ values: examples from Animal Behaviour. Animal Behaviour 56: 256-259.

Neave, H. R. and Worthington, P. L. (1988). Distribution-free tests. Unwin Hyman Ltd., London.

Odiase, J. I. and Ogbonmwan, S.M. (2005a). An algorithm for generating unconditional exact permutation distribution for a two-sample experiment. Journal of Modern Applied Statistical Methods 4(1): 319-332.

Odiase, J. I. and Ogbonmwan, S. M. (2005b). Exact permutation critical values for the Kruskal-Wallis One-Way ANOVA. Journal of Modern Applied Statistical Methods 4(2): 609-620.

Pesarin, F. (2001). Multivariate permutation tests. Wiley, New York.

Scheffe, H. (1943). Statistical inference in the nonparametric case. The Annals of Mathematical Statistics 14: 305-332.

Siegel, S. and N. J. Castellan, 1988. Nonparametric statistics for the behavioural sciences (3rd ed.). McGraw-Hill, New York. 